Document downloaded from:

http://hdl.handle.net/10251/85373

This paper must be cited as:

Mocholí Salcedo, A.; Arroyo Núñez, JH.; Milián Sánchez, VM.; Verdú Martín, GJ.; Arroyo Nuñez, A. (2017). Traffic Control Magnetic Loops Electric Characteristics Variation Due to the Passage of Vehicles Over Them. IEEE Transactions on Intelligent Transportation Systems. 18(6):1540-1548. doi:10.1109/TITS.2016.2612579.

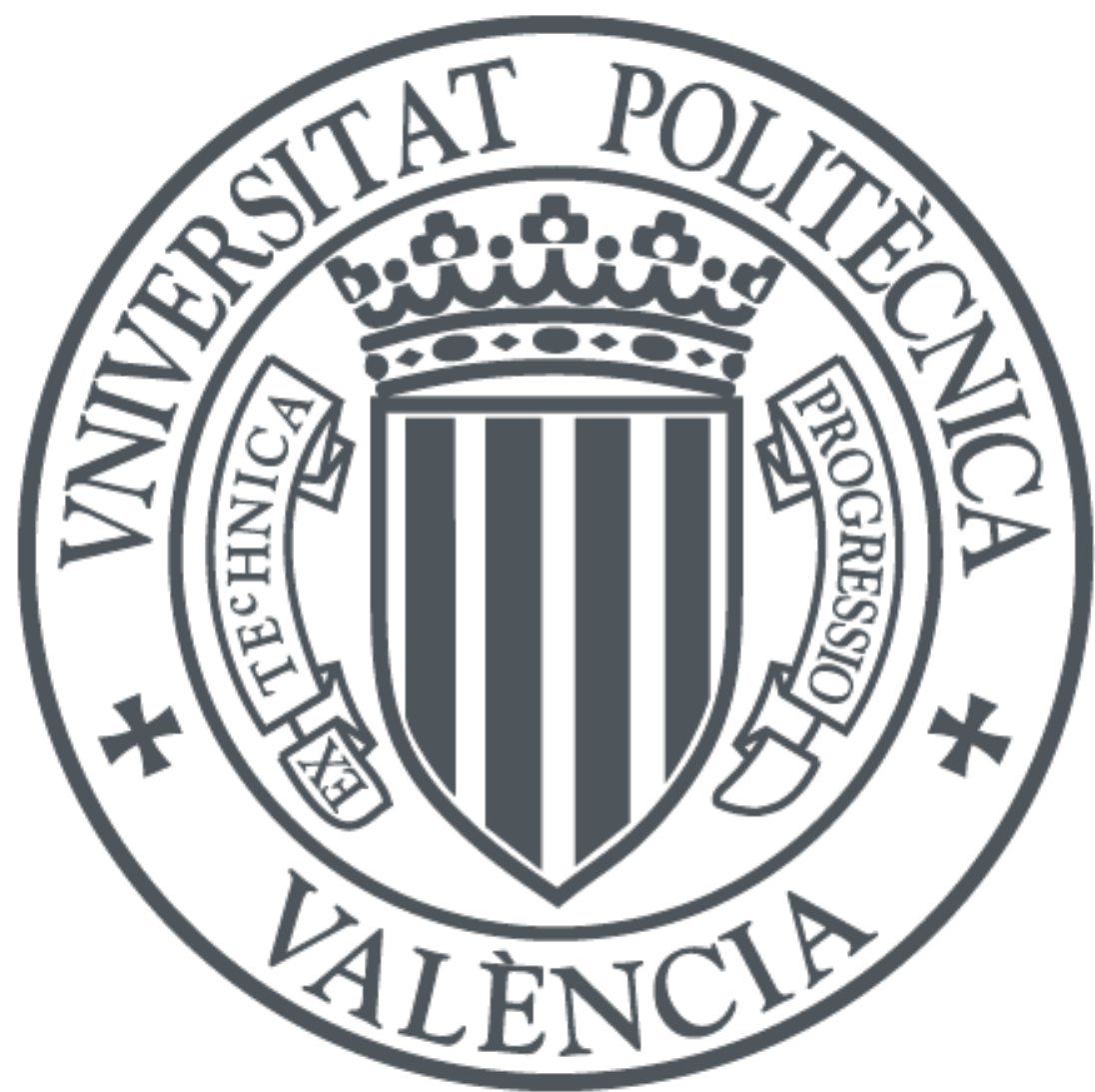

The final publication is available at

http://dx.doi.org/10.1109/JPHOT.2016.2550323

Copyright Institute of Electrical and Electronics Engineers (IEEE)

Additional Information

"(c) 2017 IEEE. Personal use of this material is permitted. Permission from IEEE must be obtained for all other users, including reprinting/ republishing this material for advertising or promotional purposes, creating new collective works for resale or redistribution to servers or lists, or reuse of any copyrighted components of this work in other works." 


\title{
Traffic Control Magnetic Loops Electric Characteristics Variation Due to the Passage of Vehicles Over Them
}

\author{
Antonio Mocholí-Salcedo, J. Humberto Arroyo-Núñez, Víctor Milián-Sánchez, Gumersindo Verdú, Alexander \\ Arroyo-Núñez
}

\begin{abstract}
From previous studies, mathematical models of magnetic fields generated by different kinds of magnetic loops are known, as well as the high correlation between those models and the real values. On this occasion, we intend to determine the effect on the buried magnetic loop response when vehicles pass over them. Specifically, a theoretical model which reflects the values of the magnetic induction in the loop is presented, as well as the changes it undergoes due to mutual induction phenomena occurring between the loop and the vehicle, when the later passes over the former. The theoretical values are compared with the actual measurements and the accuracy of the model is checked. Besides, we present a new oscillator circuit; a component being the loop. This circuit has outstanding advantages in comparison with the currently existing ones. Finally, this induction change is used to cause the oscillation frequency modification of a resonant circuit, which in turn is one of the changes used for vehicles detection, identification and classification. A program is designed and used to obtain the magnetic profile of several vehicles when passing over a rectangular loop.
\end{abstract}

Index Terms - Inductance in rectangular coils, inductive communication, magnetic field, magnetic profile, numerical calculation, vehicle detection.

\section{INTRODUCTION}

\section{A. Vehicle electrical model}

VEHICLES' detection and classification is one of the main aspects of traffic control. There exist several systems based on different phenomena which are described in the literature, together with their advantages and drawbacks [1, 2]. Some of them are video-detection [3], radio-detection [4] and magnetic detection [1]. But since their introduction in the 1960s, the most popular method is inductive loops, which provide the magnetic profile of the vehicles passing over them [5,6]. This type of vehicle detection is highly reliable and it is considered the main detection system [7, 8]. Aside from its application for vehicle's classification, which includes bicycles [9, 10, 11, 12, 13], magnetic loops can also be used for vehicle's speed measurements $[14,15$, 16], for wheels detection [17], for bi-directional communication between vehicles and infrastructures [18], or for vehicle's re-identification [19].

As previous works of different authors have highlighted, a vehicle passing over a loop can be considered as a horizontal platform [7]. This is represented in Fig. 1, where the vehicle is modeled as a rectangular plate, whose width and length equals the vehicle's respective width and length.

Fig. 1 Model of a vehicle passing over a loop. The diagrams depicted above represent the electrical model of a vehicle passing over a loop which in turn is represented in the diagram below.
A conducting mesh can be used to approach the electrical characteristics of the conducting plate. When the mesh is placed symmetrically on the inductive loop (situation in which the maximum sensibility is achieved), all internal mesh currents are canceled. This originates a unique bond of induced current which flows around the perimeter of the mesh, which in turn, is equivalent to a single rectangular short-circuited loop.

The air core transformer, depicted on the right of Fig. 1 , shows the coupling model between the vehicle chassis (represented by a turn in short circuit) and the magnetic loop placed on the road. The maximum detection sensitivity of the vehicle will take place when the distance between the two equivalent coils is minimal.

From what it is said above, it follows that the ideal inductive loop detector should have a shape that approximates the periphery of the vehicle. That is, it is preferable a square loop of $1.8 \times 1.8 \mathrm{~m}$ than just one that has the size of the vehicle's engine [7].

This model also explains the difficulty of to detect large trucks with a high trailer. The detection of these vehicles is maximized when the coil width equals the width of the truck. Another consideration to keep in mind is that the loop length should not be less than its width in order to avoid a loss of sensitivity.

However, all these studies have been performed considering a static analysis, where the vehicle was placed in a certain position with regard to the buried loop. Also, one interesting point is that with this system, the magnetic signature of any car passing over the loop can be obtained. Assuming that the speed of the vehicle is constant during the period of time it is crossing the detection zone, this magnetic signature is a signal which depends on the vehicle's position over the loop, and consequently on the time. It is precisely this (the system temporal behavior modeling), what will be analyzed in this work.

Antonio Mocholí-Salcedo is with the Traffic Control Systems Group, ITACA Institute, Universitat Politècnica de València, Camino de Vera, s/n, Valencia, Spain (e-mail: amocholi@eln.upv.es). J.Humberto Arroyo-Nuñez is with the Electronic and Telecommunications Engineering Department, Polytechnic University of Tulancingo, C/ Ingenierías no.100, Col Huapalcalco, Tulancigo de Bravo, Hidalgo, México (e-mail: humberto.arroyo@upt.edu.mx). Víctor Milián-Sánchez is with the Institute for Industrial, Radiological and Environmental Safety, Universitat Politècnica de València, Camino de Vera, s/n, Valencia, Spain (e-mail: vicmisan@iqn.upv.es ). Gumersindo Verdú-Martín, is with the Chemical and Nuclear Engineering Department, Universitat Politècnica de València, Camino de Vera, s/n, Valencia, Spain (e-mail: gverdu@iqn.upv.es). Alexander Arroyo-Nuñez is with the Biomedical Engineering Department, Polytechnic University of Chiapas, Carretera Suchiapa-Villaflores Km. 1.5 entre el Panteón y Puente Santo Domingo C.P. 29150 Suchiapa, Chiapas. México (e-mail: arroyo@upchiapas.edu.mx). 
In this study, it is assumed that the loops' inductive characteristics (of both the loop in the pavement and the loop representing the vehicle), remain constant in any relative position between the loop and the vehicle, and it is considered that the variable that varies is the mutual inductance between them. Therefore, it will be necessary to obtain a static mathematical model of the buried loop, similar to the one used in Appendix A of [7], a static model representing the vehicle similar to the one represented in Appendix E of [7] and a dynamic model for the coupling between both loops, similar to the one presented in [10]. Similar studies have been the subject of major research projects [12].

In Section II, the theory is presented and the theoretical calculations of both the inductance of a rectangular loop and the mutual inductance are respectively performed. In Section III, the various experimental setups are described along with the new hardware and software of our own development. The validation of this system is also presented. In Section IV, the results and discussion are presented and in Section V, the conclusions are outlined.

\section{THEORY}

\section{A. Inductance of a rectangular magnetic loop}

As shown in the literature [7], the inductance of a rectangular loop is given by:

$$
\mathrm{L}=\frac{\mathrm{N} \cdot \emptyset}{\mathrm{I}}
$$

where:

$\mathrm{L}=$ Inductance in Henries

$\mathrm{N}=$ Number of turns in the loop

$\varnothing=$ Magnetic flux in Webers

$\mathrm{I}=$ Current intensity in the loop in Amperes

The magnetic flux is given by:

$$
\emptyset=\int_{S} B \cdot d S,
$$

where:

$\mathrm{B}=$ Magnetic field crossing the loop

$\mathrm{S}=$ Surface through which the flux is being determined (in this case, the coil).

The magnetic field of interest to us is only the component perpendicular to the plane where the coil is located. This component is $\mathrm{B}_{\mathrm{k}}$, which is generated by the current $I$ in the loop (Fig. 2). Its value in any point of space has been determined and tested in previous works [20, 21, 22].

Fig. 2 Magnetic loop and space point where the magnetic field is analyzed.

Specifically, as shown in [21, 22], $\mathrm{Z}$ axis component is given by:

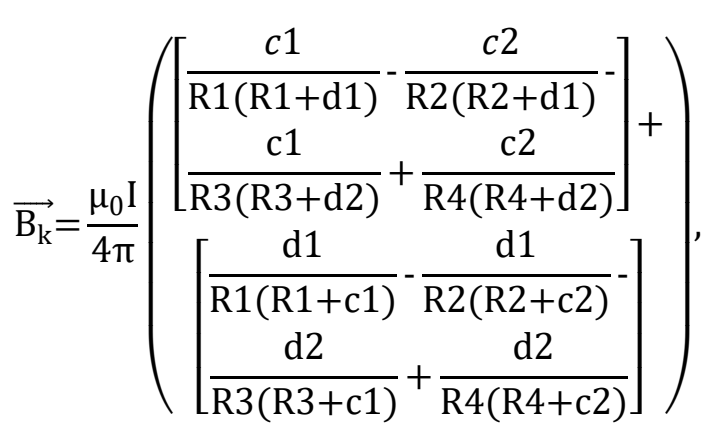

where:

$$
\begin{gathered}
R 1=\sqrt{(x+a)^{2}+(y+b)^{2}+z^{2}}=\sqrt{c 1^{2}+d 1^{2}+z^{2}} \\
R 2=\sqrt{(x-a)^{2}+(y+b)^{2}+z^{2}}=\sqrt{c 2^{2}+d 1^{2}+z^{2}} \\
R 3=\sqrt{(x+a)^{2}+(y-b)^{2}+z^{2}}=\sqrt{c 1^{2}+d 2^{2}+z^{2}} \\
R 4=\sqrt{(x-a)^{2}+(y-b)^{2}+z^{2}}=\sqrt{c 2^{2}+d 2^{2}+z^{2}} \\
c 1=-a-x \\
c 2=a-x \\
d 1=-b-y \\
d 2=b-y
\end{gathered}
$$

This Equation has been compared with the corresponding presented by Misakian [23].

The calculation of the inductance can be performed through the determination of the flux as the result of the numerical integral shown in (2) and substituting it into (1), or through the use of some of the expressions obtained as approximations to this expression [9, 18]. Among them, one of the most common Equations is [24, 25]:

$$
\mathrm{L}_{\mathrm{rect}} \approx \mathrm{N}^{2} \frac{\mu_{0} \mu_{\mathrm{r}}}{\pi}\left[\begin{array}{c}
-4(\mathrm{a}+\mathrm{b})+4 \sqrt{\mathrm{a}^{2}+\mathrm{b}^{2}}- \\
2 \mathrm{~b} \ln \left(\frac{\mathrm{b}+\sqrt{\mathrm{a}^{2}+\mathrm{b}^{2}}}{\mathrm{a}}\right)- \\
2 \mathrm{a} \ln \left(\frac{\mathrm{a}+\sqrt{\mathrm{a}^{2}+\mathrm{b}^{2}}}{\mathrm{~b}}\right)+ \\
2 \mathrm{~b} \ln \left(\frac{4 \mathrm{~b}}{\mathrm{r}}\right)+2 \mathrm{a} \ln \left(\frac{4 \mathrm{a}}{\mathrm{r}}\right)
\end{array}\right],
$$

where:

$\mu_{0}=$ free space permeability

$\mu_{\mathrm{r}}=$ medium relative permeability

$\mathrm{N}=$ number of turns of the loop

$\mathrm{r}=$ conductor radius 
$2 \mathrm{a}=$ loop length

$2 \mathrm{~b}=$ loop width.

Various calculations were performed using the two methods to determine the inductance, and it was proven that when working in the numerical integral with a number of points equal to [21, 22]:

$$
\mathrm{NP}_{\mathrm{LX}}=\frac{2 \mathrm{a}}{3 \mathrm{r}}, \quad \mathrm{NP}_{\mathrm{LY}}=\frac{2 \mathrm{~b}}{3 \mathrm{r}},
$$

the difference between the inductance values obtained with the two methods is less than $0.5 \%$.

It can be seen in (3) and (4) that a magnetic material (the vehicle), with a relative magnetic permeability higher than 1, increases the loop's inductance. The highest increase occurs when a metallic core (the vehicle) passes directly across the loop. This effect is known as the ferromagnetic effect.

In spite of the ferromagnetic effect produced by the passage of the metal mass of the vehicle (the engine, the transmission or the differential), it can be verified that the effect on the control circuit of the coil does not correspond to that effect. This is because the insertion of an iron core in the magnetic field of the inductor reduces the reluctance of the flux path, which increases the net inductance. However, the metallic perimeter of the vehicle causes an opposite effect on the inductance due to the onset of eddy currents. It can be seen in [7] that the decrease in inductance produced by eddy currents exceeds the effect of increase in inductance due to the passage of the metal mass of the vehicle, which makes that the overall net effect is a reduction in the inductance of the coil.

\section{B. Mutual inductance}

The inductance (or self-inductance) of a rectangular loop has just been obtained. When the magnetic flux of one coil is coupled to a vehicle, the coupled flux is used to obtain the mutual inductance.

In Fig. 1, the magnetic coupling between a loop and a conductor that forms a closed loop is shown; this functions like a transformer with an air core.

The mutual inductance between the primary circuit (the loop) and the secondary circuit (the conductor in form of closed loop) is given by [22]:

$$
M_{21}=\frac{N_{2} \cdot \emptyset_{21}}{I_{1}}
$$

where:

$M_{21}=$ Mutual inductance between the primary circuit and the secondary circuit, in Henries.

$N_{2}=$ Number of turns of the secondary circuit (it is 1 in case of a conductor in the shape of a closed loop).

$\emptyset_{21}=$ Magnetic flux perpendicular to the surface formed by the closed loop, in Webers.

$I_{1}=$ Current flowing through the coil, in Amperes.

\section{Global loop impedance}

When considering the combined effect of the loop embedded in the pavement and the vehicle passing over it, we should apply a model that represents this behavior. In our case, we used one of the models shown in Appendix E in [7]. According to this model, the circuit equivalent impedance (when the vehicle is passing on the loop) is given by:

$$
Z_{1}=\frac{Z_{11} Z_{22}-Z_{21}^{2}}{Z_{22}}
$$

where:

$\mathrm{Z}_{1}=$ Total equivalent impedance.

$\mathrm{Z}_{11}=$ Impedance of the loop located in the pavement and supposedly isolated.

$\mathrm{Z}_{22}=$ Impedance of the loop that represents the vehicle; also supposedly isolated.

$\mathrm{Z}_{21}=$ Mutual inductance between the loop in the pavement and the loop which represents the vehicle.

For the model under consideration, all impedances are inductances, either self-inductances or mutual inductances. Consequently, the global result is an inductance.

$\mathrm{Z}_{11}$ represents the inductance of the loop buried in the driveway when considered in isolation and without any vehicle in the vicinity. Its value is given by (1) and can be represented by $L_{e}$.

$\mathrm{Z}_{22}$ is the inductance of the loop which represents the vehicle and which can be represented by an Equation similar to (4); in this Equation, $2 \mathrm{a}$ and $2 \mathrm{~b}$ are the length and width of the vehicle, respectively, $r$ is the thickness of the metal sheet with which the vehicle's chassis has been built, and $\mathrm{N}$ is the number of turns of the loop ( 1 in this case). It is represented by $L_{v}$.

Finally, $Z_{21}$ represents the mutual inductance between the loop in the pavement and the loop that models the presence of the vehicle. Equation (6) represents this mutual inductance and can be denoted by $M_{e / v}$.

Consequently, the loop equivalent circuit is an inductance whose value is given by:

$$
L_{e q}=\frac{L_{e} L_{v}-M_{e / v}^{2}}{L_{v}}
$$

\section{MATERIALS AND METHODS}

In order to check the presented model correctness, a testing system that allowed to obtain the actual values of the buried loop equivalent inductance when a vehicle passes over it was built. In what follows the experimental setup is presented.

\section{A. Control card}

The used hardware is the system SCT-IL v2.0, which was entirely developed by the Traffic Control System Group of the Polytechnic University of Valencia (patent number P200401111). This hardware can be seen in Fig. 3. 
One of its most outstanding characteristics is that it can work simultaneously with four loops.

Fig. 3 Electronic board.

This circuit has four well differentiable parts with different applications. The hardware for control purposes is formed by two DSPs, each one for specific tasks. The software on the PC, which can be modified or changed by the user and it is also intended for the user to visualize data on a graphic interface, is user friendly and easily understandable. With this card, the system performance can be supervised in real time, and vehicles' data can be stored for further analysis.

Different models of loops were prepared and used both in the laboratory and on the public road. The loops used in the laboratory allowed to tune the functioning of the electronic circuitry. The models of loops which were tested on the public roads were used in three locations. The first was placed in the campus of the Polytechnic University of Valencia (UPV). The second was tested in Berlin and the third was located in one street of Valencia City, Spain (in Carrera Malilla Street). Each one of these places has different characteristics: type of traffic, type of road, vehicles' speeds and the types of loops were different. All the loops were buried under an asphalt layer and sealed with asphalt resin.

The first field test was performed at the university campus. The loops consisted of two small loops $(2 \times 1 \mathrm{~m}$ dimensions) and two bigger loops (dimensions $2 \times 2 \mathrm{~m}$ ). The first big loop has three turns and the second has two turns. The smaller ones have five and four turns respectively.

The traffic in the university campus is mainly constituted by small passengers' cars and occasionally by some small vans.

To gather the information, it is necessary to process the data obtained from four different graphs (one graph per each loop) like the ones shown in Fig. 4:

Fig. 4 Filtered samples obtained by the four loops.

The second test was in Berlin, specifically at the DLR facilities (Deutsches Zentrum für Luft- und Raumfahrt E.v.). Measurements were taken in two places. The electronic devices installed in each place were different and connected to two loops. These loops were of the same dimensions as the small loops at the UPV.

Finally, the third test was in Valencia City. Data gathered in the aforementioned street were used as the reference data base. This location is a two-way street; a loop in each way was installed. Each loop has three turns, dimensions $2 \times 2 \mathrm{~m}$ and has an inductance of about $84 \mu \mathrm{H}$. These sensors are property of the City Council and are intended to count the number of vehicles, but as a result of an agreement between the Council and the UPV, those installations were used for this study.

Along this street there is a flow of cars, vans, trucks and buses, and therefore, it is a very appropriate location to perform measurements and test the system functioning.

Vehicles' speed was measured with a traffic detector, manufactured by ASIM Company, model Tri-Tech TT293.
It fitted perfectly well to the requested conditions. It was installed on the post of the traffic light, at $6 \mathrm{~m}$ height.

As mentioned above, the information obtained in this location was used to create a data base. Data were obtained from the radar, from the magnetic loop, and from a laserscanner SICK LMS 221. Besides, a video-camera filmed all the sequences and a webcam recorded automatically all the vehicles' images. This data base could be used to perform all the necessary analyses without the need to travel to the test site. With this data base, different signal processing systems can be prepared and trained for applications of vehicle classification and to measure their speed and flow direction.

\section{B. Oscillator Circuit}

There are a number of circuits that can be used for the construction of an oscillator circuit based on inductances. One of the most outstanding is shown in Fig. 5, which was designed by us. It is one of the simplest and the one that offers the most advantages: specifically, it generates a digital signal and it can be easily adjusted by changing only a resistor.

As it can be noticed, the construction of the circuit requires a very small number of components. The digital output is very advantageous, as it can be used directly by microprocessor systems to process the information based on the frequency of the generated signal.

Figure 5a shows that the circuit is based on a NAND Trigger Schmidt gate and a feedback that includes the inductance. A transistor has been added to increase the current supplied to the coil.

The CONTROL input allows the activation of the oscillator. This happens when a voltage $+\mathrm{V}_{\mathrm{cc}}$ is supplied to this input. When this input is $0 \mathrm{~V}$ the oscillator is blocked and the output remains at $+\mathrm{V}_{\mathrm{cc}}$.

Fig. 5 a) Oscillator circuit. b) Oscillator circuit answer as a function of the control signal. c) Oscillator circuit answer representation when the control signal is activated.

Figure $5 \mathrm{~b}$ shows the signal generated by the circuit when the CONTROL signal changes between $0 \mathrm{~V}$ and $+\mathrm{V}_{\mathrm{cc}}$ with a cadence that allows to work sequentially with up to four loops and without any interference between them. Finally, Fig. 5c is a detail of the signal shown in Fig. 5b when the CONTROL signal is $+\mathrm{V}_{\mathrm{cc}}$.

As it can be seen in Fig. 5b and Fig. 5c, when the oscillating mode is activated (CONTROL signal at $V_{c c}$ ) the output is a square signal with values changing between $+\mathrm{V}_{\mathrm{cc}}$ and $0 \mathrm{~V}$.

We denote by $\mathrm{V}_{\mathrm{IH}}$ the lowest voltage, which is interpreted as a high input to the NAND gate, and $\mathrm{V}_{\mathrm{IL}}$ is the highest voltage, which is interpreted as the low input to the NAND gate. The output switches from 0 to $+\mathrm{V}_{\mathrm{cc}}$ when the (diminishing) input voltage to the NAND gate reaches the voltage $\mathrm{V}_{\mathrm{IL}}$. Conversely, the output switches from $+\mathrm{V}_{\mathrm{cc}}$ to 0 when the (increasing) input voltage to the NAND gate reaches the voltage $\mathrm{V}_{\mathrm{IH}}$.

When the output is $+\mathrm{V}_{\mathrm{cc}}$, the intensity along both the coil and resistor is: 


$$
I_{V c c}=\frac{V_{c c}-V_{B E}}{R}+\frac{V_{I L}-V_{c c}+V_{B E}}{R} e^{-t / \tau}
$$

where:

$\mathrm{I}_{\mathrm{Vcc}}=$ current along the feedback circuit when the output is $+\mathrm{V}_{\mathrm{cc}}$.

$\mathrm{V}_{\mathrm{BE}}=$ voltage between base-emitter in transistor (approximately 0,6 V).

$\tau=L / R$ circuit time constant which is $L_{e q} / R$.

The voltage at the NAND gate input is:

$$
\begin{aligned}
V_{I N A N D}=V_{c c}- & V_{B E}+\left(V_{I L}-V_{c c}\right. \\
& \left.+V_{B E}\right) e^{-t / \tau}
\end{aligned}
$$

The time taken by $\mathrm{V}_{\text {INAND }}$ to reach $\mathrm{V}_{\mathrm{IH}}$, point where the gate switches, is:

$$
t_{\text {on-off }}=-\tau \ln \left(\frac{V_{I H}+V_{B E}-V_{c c}}{V_{I L}+V_{B E}-V_{c c}}\right)
$$

When the output is $0 \mathrm{~V}$ the Equation governing the current along the coil and the resistor is:

$$
I_{0 V}=\frac{V_{I H}}{R} e^{-t / \tau}
$$

The voltage at the input of the NAND gate is:

$$
V_{I N A N D}=V_{I H} e^{-t / \tau}
$$

The time that takes $\mathrm{V}_{\text {INAND }}$ until it reaches $\mathrm{V}_{\text {IL }}$ (i.e., the voltage at which the switching takes place), is:

$$
t_{o f f-o n}=-\tau \ln \left(\frac{V_{I L}}{V_{I H}}\right)
$$

Consequently, the circuit oscillating frequency is:

$$
\begin{aligned}
& f=\frac{1}{t_{\text {on-off }}+t_{\text {off-on }}} \\
& =\frac{-1}{\tau} \frac{1}{\ln \left(\frac{V_{I H}+V_{B E}-V_{c c}}{V_{I L}+V_{B E}-V_{c c}}\right)+\ln \left(\frac{V_{I L}}{V_{I H}}\right)} \\
& =\frac{k}{L_{e q}}
\end{aligned}
$$

From (15) it can be deduced that the oscillation frequency is inversely proportional to the coil inductance, and the proportionality factor is a constant $k$, which is:

$$
k=\frac{-R}{\ln \left(\frac{V_{I L}}{V_{I H}}\left(\frac{V_{I H}+V_{B E}-V_{c c}}{V_{I L}+V_{B E}-V_{c c}}\right)\right)}
$$

\section{RESULTS AND DISCUSSION}

\section{A. Oscillator circuit response analysis when a vehicle passes over a loop}

In previous paragraphs, some aspects which affect the functioning of the systems using magnetic loops for vehicles detection have been analyzed. In this Section, all the calculations are applied to a real case and the theoretical and experimental values are compared.

To this end, a VisualBasic program was developed in order to carry out all the calculations (Fig. 6). The input data are the following loop parameters:

- Half side, taken on X axis: a (m)

- Half side, taken on Y axis: $b(\mathrm{~m})$

- Radius of the loop copper cable (m)

- Current along the loop (A)

- Number of turns in the loop.

- Loop core relative permeability (usually 1).

The required vehicle parameters are:

- Total length on $X$ axis (m)

- Total width on Y axis (m)

- Vehicle's height on $Z$ axis, normal to the plane of the loop (m)

- Distance travelled by the vehicle, indicating the vehicle's center initial and final position according to the three axes.

- Vehicle's speed $\left(\mathrm{m} \mathrm{s}^{-1}\right)$.

Regarding the parameters' selection, the model parameters are adjustable depending on the requirements of the installation which has to be simulated. The studied cases correspond to actual ITS systems located in the E.U. The selected vehicles represent some of the most common categories which circulate in the cities: common cars, light vans and urban transport.

Regarding the oscillating circuit, the following information is required:

- Supply voltage to the circuit, $\mathrm{V}_{\mathrm{cc}}(\mathrm{V})$.

- Transistor's base-emitter voltage, $\mathrm{V}_{\mathrm{BE}}(\mathrm{V})$.

- Switching voltages $\mathrm{V}_{\mathrm{IH}}$ and $\mathrm{V}_{\mathrm{IL}}(\mathrm{V})$.

- $\quad$ Circuit resistance $(\Omega)$.

Fig. 6 Simulation results of the passage of a vehicle (Citroën AX) over a loop.

To perform numeric integrals calculations, it is necessary to enter the number of points that must be used in the process.

With all this information, the program calculates the magnetic field in every point of space by means of (3). Also, self-inductance of both the loop and the equivalent vehicle model can be calculated using (1), (2), and (4). 
From both the described trajectory and the vehicle's velocity, the mutual inductance can be obtained at all times using (6), and from these values, one can calculate the loop equivalent inductance, as shown in (8). (15).

Oscillation frequency at all time is calculated from

The program provides in every moment the values of a set of parameters, namely, self-inductances, the equivalent inductance and the oscillation frequency. All the obtained results are stored in one file and graphically represented on the display.

To verify the proper functioning of the used models, the program is executed for a series of real situations (there is enough information available about this situations).

To this end, a standard $2 \mathrm{~m} \mathrm{x} 2 \mathrm{~m}$ coil was used (half sides of $1 \mathrm{~m}$ ); it had three turns and it was built with a copper cable of $0.375 \mathrm{~mm}$ diameter (AWG 21). To calculate the numeric integrals, 40 points were taken on each axis [22]: there is no need to take a higher number of points because both loops are separated and therefore, there is no singularity on the cables. Likewise, the mutual inductance (and consequently the oscillation frequency variation) was calculated in 40 points between the initial and final position of the vehicle over the loop (more points would have increased the resolution but at the expense of a higher calculation time).

A power supply $\mathrm{V}_{\mathrm{cc}}$ of $5 \mathrm{~V}$ was applied to the oscillator circuit. The transistor $\mathrm{V}_{\mathrm{BE}}$ voltage was $0,6 \mathrm{~V}$ and $\mathrm{V}_{\mathrm{IH}}$ and $\mathrm{V}_{\mathrm{IL}}$ were respectively $1,8 \mathrm{~V}$ and $0,95 \mathrm{~V}$; circuit resistance was about $15 \Omega$.

Regarding the vehicles, three different models were considered (two Citroën models and a city bus):

- A car of $3.531 \mathrm{~m}$ length, $1.6 \mathrm{~m}$ width and $1.34 \mathrm{~m}$ height $(\mathrm{AX})$.

- A van of $3.995 \mathrm{~m}$ length, $1.64 \mathrm{~m}$ width and 1.80 $\mathrm{m}$ height $(\mathrm{C} 15)$.

- A bus of $12 \mathrm{~m}$ length, 2,5 $\mathrm{m}$ width and $3 \mathrm{~m}$ height (city bus).

In all cases it was considered that the vehicle moved in $\mathrm{X}$ axis direction and that it was centered relatively to $\mathrm{Y}$ axis.

To represent the fact that the vehicle's height changed, it was considered that the vehicle's center took different values on $\mathrm{Z}$ axis.

The measured magnetic profiles (fingerprints) of the vehicles, along with the simulated ones, are represented in Fig. 7, Fig. 8, and Fig. 9.

In these Figures, the vertical axis represents the relative frequency displacement (frequency deviation with regard to the observed maximal displacement caused by the passing vehicle), given by:

$$
\frac{f(t)-f_{0}}{\left(f(t)-f_{0}\right)_{\max }} 100
$$

where $f(t)$ is the frequency at the time $t$, while the vehicle is passing over the loop. On the other hand, $f_{o}$ is the oscillation frequency value when there is no vehicle over the loop. Finally, $\left(\mathrm{f}(\mathrm{t})-\mathrm{f}_{\mathrm{o}}\right)_{\max }$ is the oscillation frequency maximum deviation which can be observed when the vehicle passes over the loop, i.e., the maximal peak that appears when a vehicle passes over a loop, which in turn will be different for each vehicle.

The horizontal axis represents the time in seconds.

The comparison between the real and the simulated measurements was performed using a data base obtained in the test location named "Carrera de Malilla". This data base contains the magnetic profile of each vehicle, the image of each vehicle, as well as the corresponding velocities. We have also mentioned the radar's model, which also provides the information gathered by both the ultrasonic and the infrared sensors.

Fig. 7 Measured and simulated magnetic profiles of a car (Citroën AX).

Fig. 8 Measured and simulated magnetic profiles of a delivery van (Citroën C15).

Fig. 9 Magnetic profile of a local city bus.

These Figures show that the used theoretical model gives a first approximation of the magnetic loops sensors response when a vehicle passes over them. However, they also show that more elaborated models than those used so far with simple rectangular loops, have to be developed. In this regard, one can see that for short vehicles the model fits quite well the experimental values, but they are somehow different for longer vehicles like a bus.

The authors are working on vehicle models, which are constituted by several sections of different characteristics. These models are best suited to the actual behavior and the results will be presented in next papers.

Magnetic profiles due to vehicles depend mainly of the variation experienced by the mutual inductance between the buried loop and the loop which represents the vehicle, when the latter passes over the former. As seen in the references about previous works, it has been usually assumed that a vehicle can be represented by a simple metallic rectangular plate, located at the chassis height, their dimensions being the ones of the vehicle perimeter.

Passenger cars (AX) usually have the motor on the front part; this causes the metallic mass in this part to be greater than in the rest of the vehicle. This would be equivalent to a lower height in the front part, which implies a higher mutual inductance. This is why the highest peak of the magnetic profile is displaced to the beginning of the profile.

Vehicles for cargo (C15) accumulate metallic mass in the zone where the load must be greater. Therefore, the back sides of the vans yield a greater mutual inductance, and consequently the peak of the magnetic profile is generated at the end of the vehicle.

Finally, public transport vehicles have more complex platforms, thus generating magnetic profiles with multiple peaks. At the beginning and at the end, one can find those peaks that represent the vehicle axes, and at the intermediate zone, the peaks representing the transmission and sleepers can be found.

One can highlight the differences and advances proposed in this work. In this respect it should be noted that the hardware has been entirely developed by our group. This hardware allows us to register the signal (oscillation frequency) provided simultaneously by four 
independent loops; these loops are scanned at a frequency of 666 samples per second in each channel. This is an entirely new system which allows the obtaining of the oscillation frequency and the vehicle classification, as well as their velocity and direction. Our group developed both the hardware and software of the electronic unit and of the computer where the results are displayed. On the other hand, a completely new program that allows to model any kind of loop configuration and any kind of vehicle was developed; this provides high flexibility to the design task. This simulation program is very flexible, as it allows the simulation of loops of different dimensions, number of turns, vehicle's dimensions, and trajectories.

In this work, a number of interesting contributions have been presented. Among them, the following can be emphasized:

- The theoretical model for vehicle representation (which pass over magnetic loops), presented by different authors, was tested in dynamic conditions. When referring to dynamic conditions, it means that the magnetic profile obtained corresponds to the vehicle that passes over the loop at certain velocity.

- A computer program which allows to simulate the system loop-vehicle was used. In this program, all the characteristics can be adjusted. The program also calculates the loop's magnetic field, the equivalent inductance of both the vehicle and the loop and the mutual inductance between them. All these parameters were calculated by means of both different technics and theoretical models and the similarity between them was analyzed.

- The model response was compared with the actual measurements. By this means, the possibilities and limitations that the simplified model offers to represent the actual behavior have become evident. This finding has led to the proposal of more developed models.

- A very simple oscillator circuit has been presented and implemented in a real circuit. It has been built with commercial components which offer several advantages: it can be easily adjusted according to the loop characteristics and yields a digital output without having to use an additional converter. This was verified both in the laboratory and in different sites in Spain and in Germany.

- The validation of the theoretical models has been performed by means of a multi-sensorial measuring system, which was designed and implemented. With this system, it was possible to prepare a data base which includes information about the vehicles' models (which were identified by means of the images), their velocities and the magnetic profiles that were generated when passing over a standard loop of dimensions $2 \times 2$ m.

[1 ] M. Bugdol, Z. Segiet, M. Krecichwost, P. Kasperek, "Vehicle Detection System using Magnetic Sensors", Transport Problems, vol. 9, no. 1, 2014.

\section{CONCLUSIONS}

In this work, the following results have been presented:

1) An equivalent circuit model for cars passing over a loop has been presented. The model approximates the vehicle by means of a rectangular metallic foil, its dimensions being those of the vehicle and located at the height of the vehicle's average height. Also, by means of the already known approximate formulas, the vehicle's equivalent self-inductance has been calculated. This model was previously presented by other authors and its applicability to this case has been checked.

2) The mutual inductance between the loop in the pavement and the vehicle has been calculated. To this end, the magnetic field in every point of the vehicle's model has been calculated; later on, the total flux is determined and finally, the mutual inductance is obtained from (6).

3) The equivalent inductance of the buried loop (when a vehicle passes over it) was determined using the calculated self-inductances and mutual inductances. To this end (8) was used. As a consequence of these calculations, it was verified that the passage of a vehicle over a loop causes a lowering of the equivalent inductance.

4) A simple oscillator circuit, built with inductances (which represent the equivalent inductance of both the buried loop and the loop representing the vehicle and the mutual inductance), has been presented and its functioning has been analyzed to determine its oscillation frequency. The result of this analysis shows that the oscillation frequency is inversely proportional to the loop equivalent inductance. The consequence of all this is that the passage of a vehicle over a loop causes an increase in the oscillation frequency.

5) A program which allows to simulate the magnetic loop behavior when connected to an oscillator circuit was prepared. Later, measurements with different vehicles models were taken in order to compare the model and real measurements results. From this comparison it can be seen that both the theoretical and the experimental results are very similar. Therefore, the model is good enough and can be used to perform experiments for the calculation of the parameters for traffic characterization. However, for a better representation of how the system loop-vehicle behaves, it is necessary to work with more complex vehicle models, and not only with the simple rectangular plate as it is usually done.

\section{ACKNOWLEDGMENT}

The authors wish to thank the City Council of Valencia (Spain) and the company ETRA I+D S.A. for granting this work.

\section{REFERENCES}

[2 ] L. E. Y. Mimbela, L. A. Klein, P. Kent, J. L. Hamrick, K. M. Luces, S. Herrera, "Summary of vehicle detection and surveillance technologies used in 
intelligent transportation systems", The Vehicle Detector Clearinghouse, Aug. 31, 2007, http://www.nmsu.edu/ traffic/

[3 ] J. Piecha, "Digital camera as a data source of its solution in traffic control and management", Transport Problems, vol. 7, no. 4, pp. 57-70, 2012.

[4 ] H.-J. Cho and M.-T. Tseng, "A support vector machine approach to CMOS-based radar signal processing for vehicle classification and speed estimation," Math. Comput. Modelling, vol. 58, no. 1/2, pp. 438-448, Jul. 2012.

[5 ] J. Gajda, R. Sroka, M. Stencel, A. Wajda, T. Zeglen, "A Vehicle Classification Based on Inductive Loop Detectors," IEEE Instrumentation and Measurement, Tech. Conf., Budapest, Hungry, pp. 21-23, May 2001.

[6 ] E. Minge, S. Petersen, H. Weinblatt, B. Coifmann, and E. Hoekmann, "Loop- and Length-Based Vehicle Classification, Federal Highway Administration Pooled Fund Program [TPF-5(192)]," Minnesota Department of Transportation Research Services, 395 John Ireland Boulevard, MS 330, St. Paul Minnesota 55155.

[7 ] L. A. Klein, D.R.P. Gibson, and M.K. Mills, "Traffic Detector Handbook," FHWAHRT-06-108. Federal Highway Administration, U.S. Department of Transportation 2006.

[8 ] R. L. Anderson. "Electromagnetic loop vehicle detectors," IEEE Trans. Veh. Tech., VT-19:23-30, Feb. 1970.

[9 ] A. Arroyo and A. Mocholí, "Sistemas Sensores Empleados en ITS," VII Congreso Español Sistemas Inteligentes de Transporte. Valencia, España. Sep. 2007.

[10] S. G. Burns. (2009). "In-Situ vehicle classification using an ILD and a magnetoresistive sensor array". Center for Transportation Studies, University of Minnesota, Minneapolis, Minnesota. http://www.its.umn.edu/Publications/ResearchRepor ts/reportdetail.html?id=1750.

[11] M. K. Mills, "Self-inductance formulas for quadrupole loops used with vehicle detectors," presented at the 35th IEEE VTG Conference Record, pp. 81-87, May 1985.

[12] J. V. Krogmeier, D. M. Bullock, "Inductive loop detection of bicycles and inductive loop signature processing for travel time estimation." Statewide Wireless Communications Project. vol. 2. Joint Transportation Research Program, Project No. C-36750. Purdue University. West Lafayette, Indiana 47907, Jul. 2008.

[13] J. Gajda, P. Piwowar, R. Sroka, M. Stencel, T. Zegleri, "Application of inductive loops as wheels detectors", Transp. Res. C, p. 57-66, 2012.

[14] B. R. Hellinga, "Improving freeway speed estimates from single loop detectors," J. Transp. Eng., 128, pp. 58-67, Jan./Feb. 2002.

[15] M. L. Hazelton, "Estimating vehicle speed from traffic count and occupancy data," J. of Data Sci., 2, 231-244 (2004).

[16 ] A. Tok, S. V. Hernandez, and S.G. Ritchie. "Accurate individual vehicle speeds from single inductive loop signatures," Transp. Res. Board $88^{\text {th }}$ Annual Meeting, Paper \#09-3512, 2009.
[17] J. Gajda, P. Piwowar, R. Sroka, M. Stencel, T. Zeglen, "Application of inductive loops as wheels detectors", Transp. Res. C, 21 pp. 57-66, 2012.

[18] J. H. Arroyo-Núñez, A. Mocholí-Salcedo, R. Barrales-Guadarrama and A. Arroyo-Nuñez, "Communication between magnetic loops", 16th World Road Meeting. Lisbon, Portugal, May 2010.

[19] J. Gajda, P. Burnos, "Identification of the spatial impulse response of inductive loop detectors", Int. Instrumentation and Measurement Technol. Conf. (I2MTC), 1997-2002, 2015.

[20] Z. Yu, C. Xiao, H. Wang, Y. Zhou, “The calculation of the magnetic field produced by an arbitrary shaped current-carrying wire in its plane". Proc. of the 2012 $2^{\text {nd }}$ Int. Conf. on Comput. and Inform. Appl. 2012.

[21] J.H. Arroyo-Núñez, A. Mocholí-Salcedo, I. RivasCambero, A. Arroyo-Núñez, and H. Jiménez-Mimila, "Levels of induced voltage between rectangular magnetic loops," in Proc. of the Workshop on Innovation on Information and Communication Technol. Institute ITACA, Spain, 2014, pp. 75-81. Available: http://www.tsb.upv.es/eventos/ITACAWIICT2014/WIICT2014.pdf.

[22] J. H. Humberto Núñez, "Estudio del comportamiento magnético de espiras rectangulares para la transmisión de información de corto alcance en sistemas inteligentes de transporte." Ph.D. dissertation, Dept. Elect. Eng. Universitat Politécnica de Valéncia, Valencia, Spain, 2016.

[23] M. Misakian, "Equations for the Magnetic Field Produced by One or More Rectangular Loops of Wire in the Same Plane," J. Res. Natl. Inst. Stand. Technol, vol. 105 no. 4, July-August 2000 http://www.nist.gov/jres.

[24] Technick.net, Electronic Hardware Information, Guides and Tools. Available: http://www.technick.net/public/code/cp_dpage.php? aiocp $d p=$ util_inductance rectangle.

[25] F. W. Grover, Inductance Calculations: Working Formulas and Tables, Dover Publications, Inc., 1962 p. 34 .

\begin{abstract}
About the authors:
Antonio Mocholí-Salcedo received the Ph.D. degree in Industrial Engineering from the Polytechnic University of Valencia (Universitat Politècnica de València), Spain, in 1991.

He is a Professor with the Polytechnic University of Valencia, Spain, in the Department of Electronic Engineering. $\mathrm{He}$ is also the coordinator of the Traffic Control Systems (STC) Group of the ITACA Institute at the same university.
\end{abstract}

J. Humberto Arroyo-Núñez received the M.Sc. degree in 2002 from the National Institute of Astrophysics, Optics and Electronics, México.

$\mathrm{He}$ is an assistant professor M.Sc., with the Polytechnic University of Tulancingo, México, in the Department of Electronic and Telecommunications Engineering. He is also coordinator of the Electronic and Telecommunications Engineering of the Polytechnic University of Tulancingo, México. 
Víctor M. Milián-Sánchez received the Ph.D. degree in Physics from the Polytechnic University of Valencia, Spain, in 2014.

$\mathrm{He}$ is currently an invited researcher at the Institute of Industrial, Radiological and Environmental Safety, from the Chemical and Nuclear Engineering Department at the Polytechnic University of Valencia (Universitat Politècnica de València), Spain.

Gumersindo J. Verdú-Martín received the Ph.D. degree in Industrial Engineering from the Polytechnic University of Valencia, Spain, in 1984.

$\mathrm{He}$ is a Professor with the Polytechnic University of Valencia (Universitat Politècnica de València), Spain, in the Department of Chemical and Nuclear Engineering. He is also the coordinator of the SENUBIO Group of the ISIRYM Institute at the same university.

Alexander Arroyo-Núñez received the M.Sc. degree in Electronic Engineering from the Polytechnic University of Valencia (Universitat Politècnica de València), Spain, in 2002, and he is currently working toward the Ph.D. degree in the Department of Electronic Engineering of the same university.

$\mathrm{He}$ is Director of the academic program of Biomedical Engineering at the Polytechnic University of Chiapas, México. 